\title{
Escurolles, Saint-Pont, Espinasse-Vozelle, Cognat- Lyonne, Monteignet-sur-l'Andelot, Saulzet et le Mayet-d'École
}

Jérôme Ducreux

\section{(2) OpenEdition}

\section{Journals}

Édition électronique

URL : http://journals.openedition.org/adlfi/4657

ISSN : 2114-0502

Éditeur

Ministère de la culture

Référence électronique

Jérôme Ducreux, «Escurolles, Saint-Pont, Espinasse-Vozelle, Cognat-Lyonne, Monteignet-sur-l'Andelot Saulzet et le Mayet-d'École », ADLFI. Archéologie de la France - Informations [En ligne], Auvergne, mis en ligne le 01 mars 2006, consulté le 20 avril 2019. URL : http://journals.openedition.org/adlfi/4657

Ce document a été généré automatiquement le 20 avril 2019

(c) Ministère de la Culture et de la Communication, CNRS 


\title{
Escurolles, Saint-Pont, Espinasse- Vozelle, Cognat-Lyonne, Monteignet-sur-l'Andelot, Saulzet et le Mayet-d'École
}

\author{
Jérôme Ducreux
}

Identifiant de l'opération archéologique : 145

Date de l'opération : 2006 (PI)

1 La prospection-inventaire portant sur les communes de Saulzet, Escurolles, EspinasseVozelle, Saint-Pont, Cognat-Lyonne, Monteignet-sur-l'Andelot et le Mayet-d'École a pour objectif d'inventorier et d'étudier les sites d'habitation gallo-romains observables dans ce secteur, afin d'évaluer les dynamiques d'occupation du territoire aux confins de la civitas Arvernorum,dans le cadre d'un mémoire de DEA dirigé par Frédéric Trément, professeur à l'université Blaise-Pascal de Clermont-Ferrand II.

2 La collecte des artefacts s'est faite de manière systématique, sans sélection. Toutefois, seuls les sites gallo-romains ont donné lieu à une classification typologique et à une datation fine. Les informations recensées ici sont partielles dans la mesure où la totalité des données n'a pu être traitée dans les délais imposés par la publication du BSR ; à ce titre, une notice globale sera proposée dans le BSR 2007. Nous sommes toutefois à même de produire une synthèse pour les fenêtres 1 et 2 prospectées en 2003, pour lesquelles nous avons informatisé les résultats, afin de les lier avec ceux de la campagne 2006. La carte du projet de prospection (Fig. $n^{\circ} 1$ : Cartographie des secteurs prospectés) regroupe les espaces prospectés lors des campagnes 2003 (fenêtres 1 et 2) et 2006 (fenêtres 3 à 6 ). L'espace prospecté représente $7,4 \mathrm{~km}^{2}$ environs. La prospection a été effectuée avec un maillage de $5 \mathrm{~m}$, pour un échantillonnage évalué à $40 \%$ des parcelles prospectées.

Les résultats disponibles à ce jour portent sur une surface d'environs 300 ha (fenêtres 1 et 2 hors espace non labouré). La figure 2 (Fig. $n^{\circ} 2$ : Carte de synthèse) présente une 
cartographie de la distribution des artefacts récoltés par parcelle (« unités de prospection »-UP- numérotées de 1 à 71), toutes périodes confondues. Les concentrations d'artefacts interprétées comme sites ont été pointées sur la carte, dans les parcelles concernées. À titre indicatif, nous avons également fait figurer les sites indiqués par la Carte archéologique de la Gaule, secteur Allier (sous la direction de Michel Provost, en collaboration avec Jacques Corrocher, Maurice Piboule et Monique Hilaire, Paris, 1989). Au total, sept sites ont été inventoriés et étudiés. Le site situé au nord-est de l'unité 6 correspond à un grand établissement rural occupé de la première moitié du $\mathrm{I}^{\mathrm{er}} \mathrm{s}$. apr. J.-C. jusqu'au haut Moyen Âge, IV ${ }^{e}$ s. au moins. Les sites situés dans les unités 10 et 11 sont probablement à rattacher à un même habitat rural. L'un correspond à une occupation très marquée Haut-Empire, avec présence de céramiques sigillées et de céramiques fines I ${ }^{e r}$ s.-II ${ }^{e}$ s. apr. J.-C. (unité 11). L'autre bâtiment (unité 10) correspond à une occupation Bas-Empire-haut Moyen Âge avec des caractéristiques typologiques, de taille notamment, très similaires. Il est donc fort probable qu'il s'agisse d'un déplacement d'habitat. Le site de l'unité 25 correspond à un habitat de type villa; il fournit en effet de grands vases de stockage assimilables à une activité agricole, de la vaisselle fine (sigillée, métallescente, engobée blanche, etc.), ainsi que des fragments d'hypocauste. L'occupation se situe entre le $\mathrm{I}^{\mathrm{er}} \mathrm{s}$. apr. J.-C. et le IV $\mathrm{V}^{\mathrm{e}} \mathrm{s}-\mathrm{V}^{\mathrm{e}} \mathrm{s}$. Certains tessons sporadiques de types vernis rouge pompéien et Beuvray peuvent repousser le début de l'occupation au I ${ }^{\text {er }}$ s. av. J.-C. Le site de l'unité 29 correspondrait à un bâtiment occupé depuis le $\mathrm{I}^{\mathrm{er}} \mathrm{s}$. apr. J.C. La fin de l'occupation est difficile à établir. Une phase Bas-Empire - haut Moyen Âge peut être caractérisée avec une quasi-certitude. Le site de l'unité 30 pose les mêmes problèmes de conservation des artefacts. On peut toutefois avancer une occupation s'étendant de la première moitié du $\mathrm{I}^{\mathrm{er}} \mathrm{s}$. apr. J.-C. au haut Moyen Âge.

Enfin, le site de l'unité 17 correspond à une activité médiévale, sans plus de précision.

5 Jérôme Ducreux

\section{ANNEXES}


Fig. $n^{\circ} 1$ : Cartographie des secteurs prospectés

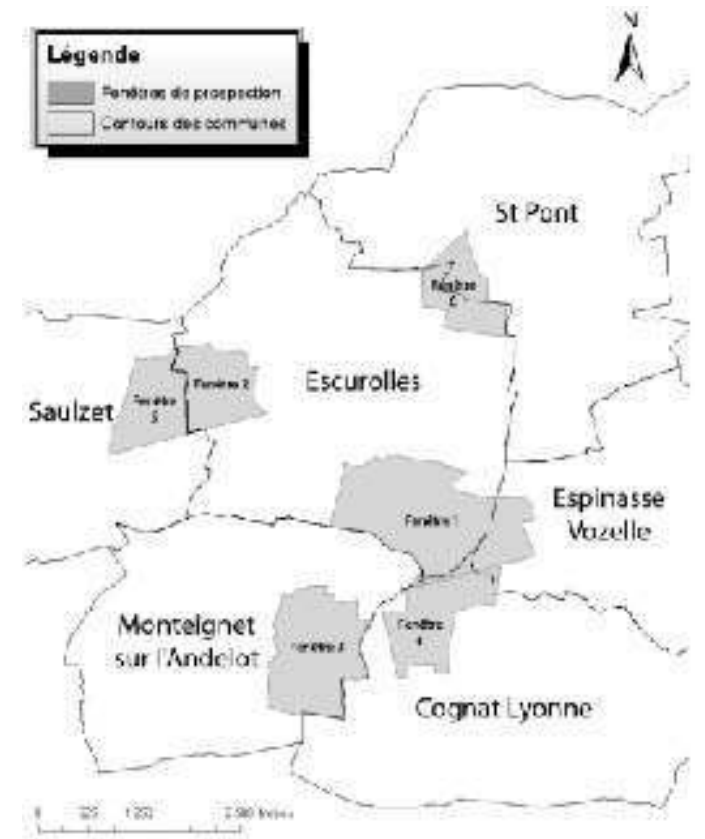

Auteur(s) : Ducreux, Jérôme (BEN). Crédits : Ducreux Jérôme, BEN (2006)

Fig. $n^{\circ} 2$ : Carte de synthèse

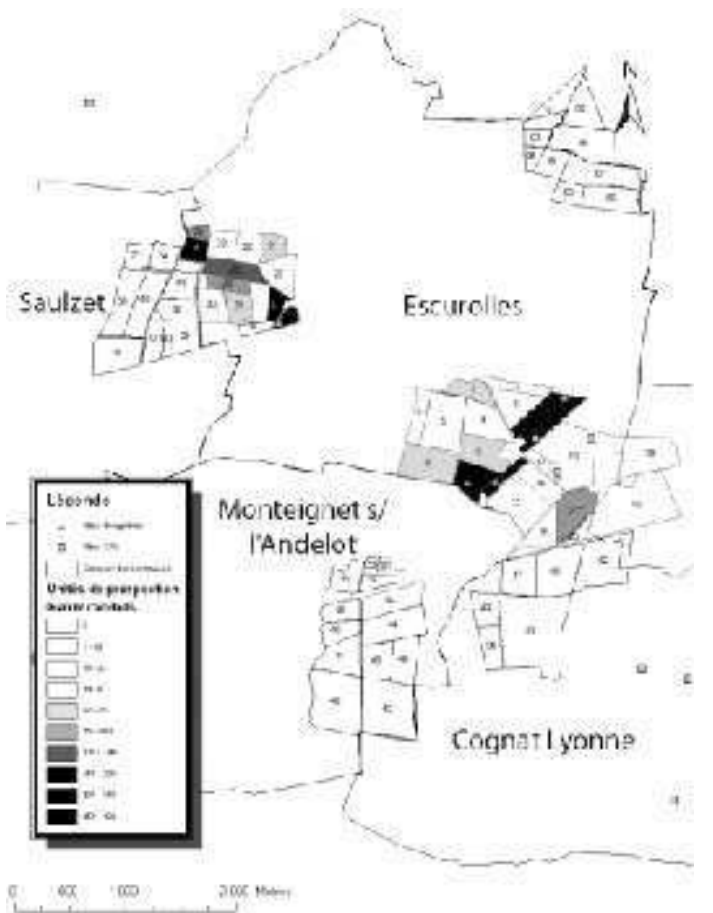

Auteur(s) : Ducreux, Jérôme (BEN). Crédits : Ducreux Jérôme, BEN (2006) 
INDEX

Index géographique : Auvergne, Allier (03), Escurolles

operation Prospection inventaire (PI)

Thèmes : agriculture, céramique gallo-romaine, habitat rural, habitation, hypocauste, sigillée, stockage, villa

Index chronologique : Empire romain, Moyen Âge

\section{AUTEURS}

JÉRÔME DUCREUX

BEN 